\title{
Differential Inequalities for One Component of Solution Vector for Systems of Linear Functional Differential Equations
}

\author{
Alexander Domoshnitsky \\ Department of Mathematics and Computer Science, The Ariel University Center of Samaria, \\ 44837 Ariel, Israel \\ Correspondence should be addressed to Alexander Domoshnitsky, adom@ariel.ac.il
}

Received 24 December 2009; Accepted 26 April 2010

Academic Editor: Ağacik Zafer

Copyright (C) 2010 Alexander Domoshnitsky. This is an open access article distributed under the Creative Commons Attribution License, which permits unrestricted use, distribution, and reproduction in any medium, provided the original work is properly cited.

\begin{abstract}
The method to compare only one component of the solution vector of linear functional differential systems, which does not require heavy sign restrictions on their coefficients, is proposed in this paper. Necessary and sufficient conditions of the positivity of elements in a corresponding row of Green's matrix are obtained in the form of theorems about differential inequalities. The main idea of our approach is to construct a first order functional differential equation for the $n$th component of the solution vector and then to use assertions about positivity of its Green's functions. This demonstrates the importance to study scalar equations written in a general operator form, where only properties of the operators and not their forms are assumed. It should be also noted that the sufficient conditions, obtained in this paper, cannot be improved in a corresponding sense and does not require any smallness of the interval $[0, \omega]$, where the system is considered.
\end{abstract}

\section{Introduction}

Consider the following system of functional differential equations

$$
\left(M_{i} x\right)(t) \equiv x_{i}^{\prime}(t)+\sum_{j=1}^{n}\left(B_{i j} x_{j}\right)(t)=f_{i}(t), \quad t \in[0, \omega], i=1, \ldots, n,
$$

where $x=\operatorname{col}\left(x_{1}, \ldots, x_{n}\right), B_{i j}: C_{[0, \omega]} \rightarrow L_{[0, \omega]}, i, j=1, \ldots, n$, are linear continuous operators, $C_{[0, \omega]}$ and $L_{[0, \omega]}$ are the spaces of continuous and summable functions $y:[0, \omega] \rightarrow$ $R^{1}$, respectively. 
Let $l: C_{[0, \omega]}^{n} \rightarrow R^{n}$ be a linear bounded functional. If the homogeneous boundary value problem $\left(M_{i} x\right)(t)=0, t \in[0, \omega], i=1, \ldots, n, l x=0$, has only the trivial solution, then the boundary value problem

$$
\left(M_{i} x\right)(t)=f_{i}(t), \quad t \in[0, \omega], i=1, l x=\alpha,
$$

has for each $f=\operatorname{col}\left(f_{1}, \ldots, f_{n}\right)$, where $f_{i} \in L_{[0, \omega]}, i=1, \ldots, n$, and $\alpha \in R^{n}$, a unique solution, which has the following representation [1]:

$$
x(t)=\int_{0}^{\omega} G(t, s) f(s) d s+X(t) \alpha, \quad t \in[0, \omega]
$$

where the $n \times n$ matrix $G(t, s)$ is called Green's matrix of problem (1.2), and $X(t)$ is the $n \times n$ fundamental matrix of the system $\left(M_{i} x\right)(t)=0, i=1, \ldots, n$, such that $l X=E$ ( $E$ is the unit $n \times n$-matrix). It is clear from the solution representation (1.3) that the matrices $G(t, s)$ and $X(t)$ determine all properties of solutions.

The following property is the basis of the approximate integration method by Tchaplygin [2]: from the conditions

$$
\left(M_{i} x\right)(t) \geq\left(M_{i} y\right)(t), \quad t \in[0, \omega], i=1, \ldots, n, l x=l y,
$$

it follows that

$$
x_{i}(\mathrm{t}) \geq y_{i}(t), \quad t \in[0, \omega], i=1, \ldots, n .
$$

Series of papers, started with the known paper by Luzin [3], were devoted to the various aspects of Tchaplygin's approximate method. The well-known monograph by Lakshmikantham and Leela [4] was one of the most important in this area. The known book by Krasnosel'skii et al. [5] was devoted to approximate methods for operator equations. These ideas have been developing in scores of books on the monotone technique for approximate solution of boundary value problems for systems of differential equations. Note in this connection the important works by Kiguradze and Puza [6, 7] and Kiguradze [8].

As a particular case of system (1.1), let us consider the following delay system:

$$
\begin{aligned}
x_{i}^{\prime}(t)+\sum_{j=1}^{n} p_{i j}(t) x_{j}\left(h_{i j}(t)\right) & =f_{i}(t), \quad i=1, \ldots, n, t \in[0, \omega], \\
x(\xi) & =0 \quad \text { for } \xi<0,
\end{aligned}
$$

where $p_{i j}$ are summable functions, and $h_{i j}$ are measurable functions such that $h_{i j}(t) \leq t$ for $i, j=1, \ldots, n, t \in[0, \omega]$. 
The classical Wazewskii's theorem claims [9] that the condition

$$
p_{i j} \leq 0 \text { for } j \neq i, i, j=1, \ldots, n \text {, }
$$

is necessary and sufficient for the property $(1.4) \Rightarrow(1.5)$ for the Cauchy problem for system of ordinary differential equations

$$
x_{i}^{\prime}(t)+\sum_{j=1}^{n} p_{i j}(t) x_{j}(t)=f_{i}(t), \quad i=1, \ldots, n, t \in[0, \omega]
$$

From formula of solution representation (1.3), it is clear that property $(1.4) \Rightarrow(1.5)$ is true if all elements of the matrices $G(t, s)$ and $X(t)$ are nonnegative.

We focus our attention upon the problem of comparison for only one of the components of solution vector. Let $k_{i}$ be either 1 or 2 . In this paper we consider the following property: from the conditions

$$
(-1)^{k_{i}}\left[\left(M_{i} x\right)(t)-\left(M_{i} y\right)(t)\right] \geq 0, \quad t \in[0, \omega], l x=l y, i=1, \ldots, n,
$$

it does follow that for a corresponding fixed component $x_{r}$ of the solution vector the inequality

$$
x_{r}(t) \geq y_{r}(t), \quad t \in[0, \omega]
$$

is satisfied. This property is a weakening of the property $(1.4) \Rightarrow(1.5)$ and, as we will obtain below, leads to essentially less hard limitations on the given system. From formula of solution's representation (1.3), it follows that this property is reduced to sign-constancy of all elements standing only in the $r$ th row of Green's matrix.

The main idea of our approach is to construct a corresponding scalar functional differential equation of the first order

$$
x_{n}^{\prime}(t)+\left(B x_{n}\right)(t)=f^{*}(t), \quad t \in[0, \omega]
$$

for $n$th component of a solution vector, where $B: C_{[0, \omega]} \rightarrow L_{[0, \omega]}$ is a linear continuous operator, $f^{*} \in L_{[0, \omega]}$. This equation is built in Section 2 . Then the technique of analysis of the first-order scalar functional differential equations, developed, for example, in the works [10-12], is used. On this basis in Section 3 we obtain necessary and sufficient conditions of nonpositivity/nonnegativity of elements in nth row of Green's matrices in the form of theorems about differential inequalities. Simple coefficient tests of the sign constancy of the elements in the $n$th row of Green's matrices are proposed in Section 4 for systems of ordinary differential equations and in Section 5 for systems of delayed differential equations. It should be stressed that in our results a smallness of the interval $[0, \omega]$ is not assumed.

Note that results of this sort for the Cauchy problem (i.e., $l x \equiv \operatorname{col}\left(x_{1}(0), \ldots, x_{n}(0)\right)$ and Volterra operators $B_{i j}: C_{[0, \omega]} \rightarrow L_{[0, \omega]}$ were proposed in the recent paper [13], where the obtained operator $B: C_{[0, \omega]} \rightarrow L_{[0, \omega]}$ became a Volterra operator. In this paper we consider other boundary conditions that imply that the operator $B: C_{[0, \omega]} \rightarrow L_{[0, \omega]}$ is not a Volterra one even in the case when all $B_{i j}: C_{[0, \omega]} \rightarrow L_{[0, \omega]}, i, j=1, \ldots, n$, are Volterra operators. 


\section{Construction of Equation for $n$th Component of Solution Vector}

In this paragraph, we consider the boundary value problem

$$
\begin{gathered}
\left(M_{i} x\right)(t) \equiv x_{i}^{\prime}(t)+\sum_{j=1}^{n}\left(B_{i j} x_{j}\right)(t)=f_{i}(t), \quad t \in[0, \omega], i=1, \ldots, n, \\
l_{i} x_{i}=c_{i}, \quad i=1, \ldots, n,
\end{gathered}
$$

where $B_{i j}: C_{[0, \omega]} \rightarrow L_{[0, \omega]}$ are linear bounded operators for $i, j=1, \ldots, n$, and $l_{i}: C_{[0, \omega]} \rightarrow$ $R^{1}, i=1, \ldots, n$, are linear boundary functionals

Together with problem (2.1), (2.2) let us consider the following auxiliary problem consisting of the system:

$$
\left(m_{i} x\right)(t) \equiv x_{i}^{\prime}(t)+\sum_{j=1}^{n-1}\left(B_{i j} x_{j}\right)(t)=f_{i}(t), \quad t \in[0, \omega], i=1, \ldots, n-1,
$$

of the order $n-1$ and the boundary conditions

$$
l_{i} x_{i}=c_{i}, \quad i=1, \ldots, n-1 .
$$

Let us assume that problem (2.3), (2.4) is uniquely solvable; denote by $K(t, s)=$ $\left\{K_{i j}(t, s)\right\}_{i, j=1, \ldots, n-1}$ its Green's matrix and by $G(t, s)=\left\{G_{i j}(t, s)\right\}_{i, j=1, \ldots, n}$ Green's matrix of the problem (2.1), (2.2).

Let us start with the following assertion, explaining how the scalar functional differential equation for one of the components of the solution vector can be constructed.

Lemma 2.1. The component $x_{n}$ of the solution vector of system (2.1) satisfies the following scalar functional differential equation:

$$
x_{n}^{\prime}(t)+\left(B x_{n}\right)(t)=f^{*}(t), \quad t \in[0, \omega],
$$

where the operator $B: C_{[0, \omega]} \rightarrow L_{[0, \omega]}$ and the function $f^{*} \in L_{[0, \omega]}$ are defined by the equalities

$$
\begin{aligned}
& \left(B x_{n}\right)(t) \equiv-\sum_{i=1}^{n-1} B_{n i}\left\{\int_{0}^{\omega} \sum_{j=1}^{n-1} K_{i j}(\cdot, s)\left(B_{j n} x_{n}\right)(s) d s\right\}(t)+\left(B_{n n} x_{n}\right)(t), \quad t \in[0, \omega] \\
& f^{*}(t)=f_{n}(t)-\sum_{i=1}^{n-1} B_{n i}\left\{\int_{0}^{\omega} \sum_{j=1}^{n-1} K_{i j}(\cdot, s) f_{j}(s) d s\right\}(t)-\sum_{i=1}^{n-1} B_{n i}\left\{\sum_{j=1}^{n-1} K_{i j}(\cdot, 0) c_{j}\right\}(t),
\end{aligned}
$$


where $u=\operatorname{col}\left\{u_{1}, \ldots, u_{n-1}\right\}$ is the solution of the system

$$
\left(m_{i} x\right)(t)=0, \quad t \in[0, \omega], i=1, \ldots, n-1,
$$

satisfying condition (2.4).

Proof. Using Green's matrix $K(t, s)=\left\{K_{i j}(t, s)\right\}_{i, j=1}^{n-1}$ of problem (2.3), (2.4), we obtain

$$
x_{i}(t)=-\int_{0}^{\omega} \sum_{j=1}^{n-1} K_{i j}(t, s)\left(B_{j n} x_{n}\right)(s) d s+\int_{0}^{\omega} \sum_{j=1}^{n-1} K_{i j}(t, s) f_{j}(s) d s+\sum_{j=1}^{n-1} K_{i j}(t, 0) c_{j},
$$

for every $i \in\{1, \ldots, n-1\}$. Substitution of these representations in the $n$th equation of the system (2.1) leads to (2.5), where the operator $B$ and the function $f^{*}$ are described by formulas (2.6) and (2.7), respectively.

\section{Positivity of the Elements in the Fixed $n$th Row of Green's Matrices}

Consider the boundary value problem

$$
\begin{gathered}
\left(M_{i} x\right)(t) \equiv x_{i}^{\prime}(t)+\sum_{j=1}^{n}\left(B_{i j} x_{j}\right)(t)=f_{i}(t), \quad t \in[0, \omega], i=1, \ldots, n, \\
l_{i} x_{i}=c_{i}, \quad i=1, \ldots, n-1, \quad x_{n}(\omega)=c_{n},
\end{gathered}
$$

where $B_{i j}: C_{[0, \omega]} \rightarrow L_{[0, \omega]}$ are linear continuous operators for $i, j=1, \ldots, n$.

Theorem 3.1. Let problem (2.3), (2.4) be uniquely solvable, all elements of its $(n-1) \times(n-1)$ Green's matrix $K(t, s)$ nonnegative, and the operators $B_{i n},-B_{n i}$ and $B_{n n}$ positive operators for $i=1, \ldots, n-1$. Then the following 2 assertions are equivalent:

(1) there exists an absolutely continuous vector function $v$ such that $v_{n}(t)>0,\left(M_{i} v\right)(t) \leq 0$, for $t \in[0, \omega], i=1, \ldots, n$, and the solution of the homogeneous equation $\left(m_{i} u\right)(t)=0$ for $t \in[0, \omega], i=1, \ldots, n-1$, satisfying the conditions $l_{i} u_{i}=l_{i} v_{i}, i=1, \ldots, n-1$, is nonpositive;

(2) the boundary value problem (3.1) is uniquely solvable for every summable $f=$ $\operatorname{col}\left(f_{1}, \ldots, f_{n}\right)$ and $c=\operatorname{col}\left(c_{1}, \ldots, c_{n}\right) \in R^{n}$ and elements of the nth row of its Green's matrix satisfy the inequalities: $G_{n j}(t, s) \leq 0$ for $j=1, \ldots, n, t, s \in[0, \omega]$, while $G_{n n}(t, s)<0$ for $0 \leq t<s \leq \omega$.

Proof. Let us start with the implication $(1) \Rightarrow(2)$. By virtue of Lemma 2.1, the component $x_{n}$ of the solution vector of problem (3.1) satisfies (2.5). Condition (1) by virtue of Theorem 1 of the paper [14] implies that Green's function $\mathrm{G}_{N}(t, s)$ of the boundary value problem

$$
x_{n}^{\prime}(t)+\left(B x_{n}\right)(t)=f^{*}(t), \quad t \in[0, \omega], x(\omega)=0,
$$


exists and satisfies the inequalities $G_{N}(t, s) \leq 0$ for $t, s \in[0, \omega]$, while $G_{N}(t, s)<0$ for $0 \leq t<$ $s \leq \omega$. Lemma 2.1, the representations of solutions of boundary value problem (3.1) and the scalar one-point problem (3.2) imply the equality

$$
x_{n}(t)=\int_{0}^{\omega} \sum_{j=1}^{n} G_{n j}(t, s) f_{j}(s) d s=\int_{0}^{\omega} G_{N}(t, s) f^{*}(s) d s, \quad t \in[0, \omega] .
$$

If $B_{n j}$ is a negative operator for every $j=1, \ldots, n-1$, and $f_{i} \leq 0$ for $i=1, \ldots, n$, then $f^{*} \leq 0$. The nonpositivity of $G_{N}(t, s)$ implies that $x_{n}$ is nonnegative and consequently $G_{n j}(t, s) \leq 0$ for $t, s \in[0, \omega]$ and $j=1, \ldots, n$.

If we set $f_{j}=0$ for $j=1, \ldots, n-1$, and $l_{j} x_{j}=0$ for $j=1, \ldots, n$, then

$$
x_{n}(t)=\int_{0}^{\omega} G_{n n}(t, s) f_{n}(s) d s=\int_{0}^{\omega} G_{N}(t, s) f_{n}(s) d s, \quad t \in[0, \omega]
$$

and it is clear that $G_{n n}(t, s)=G_{N}(t, s)$. It is known from Theorem 1 of the paper [14] that $G_{N}(t, s)<0$ for $0 \leq t<s \leq \omega$. This implies that $G_{n n}(t, s)<0$ for $0 \leq t<s \leq \omega$.

In order to prove $(2) \Rightarrow(1)$, let us define $v_{i}(1=1, \ldots, n)$ by the following way:

$$
v_{i}(t)=w_{i}(t), \quad i=1, \ldots, n-1, \quad v_{n}(t)=w_{n}(t)+1, \quad t \in[0, \omega],
$$

where $w_{i}(1=1, \ldots, n)$ is a solution to the problem

$$
w_{i}^{\prime}(t)+\sum_{j=1}^{n}\left(B_{i j} w_{j}\right)(t)=-\left(B_{\text {in }} 1\right)(t), \quad i=1, \ldots, n, t \in[0, \omega] .
$$

It is clear that the functions $v_{i}(1=1, \ldots, n)$ satisfy the homogeneous system

$$
v_{i}^{\prime}(t)+\sum_{j=1}^{n}\left(B_{i j} v_{j}\right)(t)=0, \quad i=1, \ldots, n, \quad t \in[0, \omega]
$$

and $v_{n}(t)>0$ for $t \in[0, \omega]$.

Theorem 3.2. Let problem (2.3), (2.4) be uniquely solvable, all elements of its $(n-1) \times(n-1)$ Green's matrix $K(t, s)$ nompositive, and $B_{n n},-B_{\text {in }}$ and $-B_{n i}$ positive operators for $i=1, \ldots, n-1$. Then the following 2 assertions are equivalent:

$\left(1^{*}\right)$ there exists an absolutely continuous vector function $v$ such that $v_{n}(t)>0,\left(M_{n} v\right)(t) \leq$ $0,\left(M_{i} v\right)(t) \geq 0$ for $t \in[0, \omega], i=1, \ldots, n-1$, and the solution of the homogeneous equation $\left(m_{i} u\right)(t)=0$ for $t \in[0, \omega], i=1, \ldots, n-1$, satisfying the conditions $l_{i} u_{i}=$ $l_{i} v_{i}, i=1, \ldots, n-1$, is nonnegative;

$\left(2^{*}\right)$ the boundary value problem (3.1) is uniquely solvable for every summable $f=$ $\operatorname{col}\left(f_{1}, \ldots, f_{n}\right)$ and $c=\operatorname{col}\left(c_{1}, \ldots, c_{n}\right) \in R^{n}$ and elements of the nth row of its Green's matrix satisfies the inequalities: $G_{n j}(t, s) \geq 0$ for $j=1, \ldots, n-1, G_{n n}(t, s) \leq 0$ for $t, s \in[0, \omega]$ while $G_{n n}(t, s)<0$ for $0 \leq t<s \leq \omega$.

The proof of this theorem is analogous to the proof of Theorem 3.1. 


\section{Sufficient Conditions of Nonpositivity of the Elements in the $n$th Row of Green's Matrices for System of Ordinary Differential Equations}

In this paragraph, we consider the system of the ordinary differential equations

$$
x_{i}^{\prime}(t)+\sum_{j=1}^{n} p_{i j}(t) x_{j}(t)=f_{i}(t), \quad i=1, \ldots, n, t \in[0, \omega],
$$

with the boundary conditions

$$
x_{i}(0)=x_{i}(\omega)+c_{i}, \quad i=1, \ldots, n-1, \quad x_{n}(\omega)=c_{n} .
$$

Theorem 4.1. Let the following conditions be fulfilled:

(1) $p_{i j} \leq 0$ for $i \neq j, i, j=1, \ldots, n-1$;

(2) $p_{j n} \geq 0, p_{n j} \leq 0$ for $j=1, \ldots, n-1, p_{n n} \geq 0$;

(3) there exists a positive number $\alpha$ such that

$$
p_{n n}(t)-\sum_{j=1}^{n-1} p_{n j}(t) \leq \alpha \leq \min _{1 \leq i \leq n-1}\left\{-p_{i n}(t)+\sum_{j=1}^{n-1} p_{i j}(t)\right\}, \quad t \in[0, \omega] .
$$

Then problem (4.1), (4.2) is uniquely solvable for every summable $f=\operatorname{col}\left(f_{1}, f_{2}, f_{n}\right)$ and $c=$ $\operatorname{col}\left(c_{1}, c_{2}, \ldots, c_{n}\right) \in R^{n}$, and the elements of the $n$th row of Green's matrix of boundary value problem (4.1), (4.2) satisfy the inequalities: $G_{n j}(t, s) \leq 0$ for $j=1, \ldots, n$, for $t, s \in[0, \omega], G_{n n}(t, s)<0$ for $0 \leq t<s \leq \omega$.

Proof. Let us prove that all elements of Green's matrix $K(t, s)$ of the auxiliary boundary value problem

$$
\begin{gathered}
x_{i}^{\prime}(t)+\sum_{j=1}^{n-1} p_{i j}(t) x_{j}(t)=f_{i}(t), \quad i=1, \ldots, n-1, t \in[0, \omega], \\
x_{i}(0)=x_{i}(\omega)+c_{i}, \quad i=1, \ldots, n-1,
\end{gathered}
$$

are nonnegative. The conditions (1), (2), and the inequality

$$
0<\alpha \leq \min _{1 \leq i \leq n-1}\left\{-p_{i n}(t)+\sum_{j=1}^{n-1} p_{i j}(t)\right\}, \quad t \in[0, \omega]
$$

imply that the conditions (1) and (2) of Theorem 3.1 of the paper [13] are fulfilled. Assertion (a) of Theorem 3.1 [13] is fulfilled. To prove it, we set $v_{i}=1$ for $1=1, \ldots, n-1$ in this assertion. 
Now according to equivalence of assertions (a) and (b) in Theorem 3.1 of the paper [13], we get the nonnegativity of all elements of its Green's matrix $K(t, s)$.

Let us set $v_{i}(t)=-e^{-\alpha t}$ for $i=1, \ldots, n-1$, and $v_{n}(t)=e^{-\alpha t}$ in the condition (1) of Theorem 3.1. We obtain that this condition is satisfied if $\alpha$ satisfies the following system of the inequalities:

$$
\begin{gathered}
\alpha \leq-p_{i n}(t)+\sum_{j=1}^{n-1} p_{i j}(t), \quad i=1, \ldots, n-1, t \in[0, \omega], \\
p_{n n}(t)-\sum_{j=1}^{n-1} p_{n j}(t) \leq \alpha, \quad t \in[0, \omega] .
\end{gathered}
$$

Now by virtue of Theorem 3.1, all elements of the $n$th row of Green's matrix satisfy the inequalities $G_{n j}(t, s) \leq 0$ for $j=1, \ldots, n-1$, and, using [14], we can conclude that $G_{n n}(t, s)<$ 0 for $0 \leq t<s \leq \omega$.

Consider now the following ordinary differential system of the second order;

$$
\begin{aligned}
& x_{1}^{\prime}(t)+p_{11}(t) x_{1}(t)+p_{12}(t) x_{2}(t)=f_{1}(t), \quad t \in[0, \omega] \\
& x_{2}^{\prime}(t)+p_{21}(t) x_{1}(t)+p_{22}(t) x_{2}(t)=f_{2}(t),
\end{aligned}
$$

with the conditions

$$
x_{1}(0)=x_{1}(\omega)+c_{1}, \quad x_{2}(\omega)=c_{2} .
$$

From Theorem 4.1 as a particular case for $n=2$, we obtain the following assertion.

Theorem 4.2. Let the following two conditions be fulfilled:

(1) $p_{11} \geq 0, p_{12} \geq 0, p_{21} \leq 0, p_{22} \geq 0$;

(2) there exists a positive $\alpha$ such that

$$
p_{22}(t)-p_{21}(t) \leq \alpha \leq p_{11}(t)-p_{12}(t), \quad t \in[0, \omega] .
$$

Then problem (4.7), (4.8) is uniquely solvable for every summable $f=\operatorname{col}\left(f_{1}, f_{2}\right)$ and $c=$ $\left\{c_{1}, c_{2}\right\} \in R^{2}$, and the elements of the second row of Green's matrix of problem (4.7), (4.8) satisfy the inequalities: $G_{2 i}(t, s) \leq 0$ for $1=1,2, t \in[0, \omega], G_{22}(t, s)<0$ for $0 \leq t<s<\omega$.

Remark 4.3. If coefficients $p_{i j}$ are constants, the second condition in Theorem 4.2 is as follows:

$$
p_{22}-p_{21} \leq p_{11}-p_{12}, \quad p_{11}-p_{12}>0
$$


Remark 4.4. Let us demonstrate that inequality (4.10) is best possible in a corresponding case and the condition

$$
p_{22}-p_{21} \leq p_{11}-p_{12}+\varepsilon, \quad p_{11}-p_{12}+\varepsilon>0
$$

cannot be set instead of (4.10). The characteristic equation of the system

$$
\begin{aligned}
& x_{1}^{\prime}(t)+p_{11} x_{1}(t)+p_{12} x_{2}(t)=0 \\
& x_{2}^{\prime}(t)+p_{21} x_{1}(t)+p_{22} x_{2}(t)=0
\end{aligned}
$$

with constant coefficients is as follows:

$$
\lambda^{2}+\left(p_{11}+p_{22}\right) \lambda+p_{11} p_{22}-p_{12} p_{21}=0
$$

If we set $p_{11}=p_{22}=0, p_{21}<0, p_{12}>0, p_{12}-p_{21}<\varepsilon$, then the roots are $\lambda_{1}=i \sqrt{-p_{12} p_{21}}$, $\lambda_{2}=-i \sqrt{-p_{12} p_{21}}$, and the problem

$$
\begin{gathered}
x_{1}^{\prime}(t)+p_{12} x_{2}(t)=0, \quad t \in[0, \omega], \\
x_{2}^{\prime}(t)+p_{21} x_{1}(t)=0, \\
x_{1}(0)=x_{1}(\omega), \quad x_{2}(\omega)=0
\end{gathered}
$$

has nontrivial solution for $\omega=2 \pi / \sqrt{-p_{12} p_{21}}$.

\section{Sufficient Conditions of Nonpositivity of the Elements in the $n$th Row of Green's Matrices for Systems with Delay}

Let us consider the system of the delay differential equations

$$
\begin{gathered}
x_{i}^{\prime}(t)+\sum_{j=1}^{n} p_{i j}(t) x_{j}\left(t-\tau_{i j}(t)\right)=f_{i}(t), \quad i=1, \ldots, n, t \in[0, \omega] . \\
x_{i}(\xi)=0 \text { for } \xi<0, i=1, \ldots, n,
\end{gathered}
$$

with the boundary conditions

$$
x_{i}(0)=x_{i}(\omega)+c_{i}, \quad i=1, \ldots, n-1, x_{n}(\omega)=c_{n} .
$$

We introduce the denotations: $p_{i j}^{*}=\operatorname{ess} \sup p_{i j}(t), p_{i j *}=\operatorname{ess} \inf p_{i j}(t), \tau_{i j}^{*}=$ ess $\sup \tau_{i j}(t)$, and $\tau_{i j *}=\operatorname{ess} \inf \tau_{i j}(t)$. 
Theorem 5.1. Let the following conditions be fulfilled:

(1) $p_{i j} \leq 0$ for $i \neq j, i, j=1, \ldots, n-1$;

(2) $p_{j n} \geq 0, p_{n j} \leq 0$ for $j=1, \ldots, n-1, p_{n n} \geq 0$;

(3) $\tau_{i i}=0$ for $i=1, \ldots, n-1$;

(4) there exists a positive number $\alpha$ such that

$$
\begin{aligned}
& p_{n n}(t) e^{\alpha \tau_{n n}(t)}-\sum_{j=1}^{n-1} p_{n j}(t) e^{\alpha \tau_{n j}(t)} \\
& \quad \leq \alpha \leq \min _{1 \leq i \leq n-1}\left\{-p_{i n}(t) e^{\alpha \tau_{i n}(t)}+p_{i i}(t)+\sum_{j=1, i \neq j}^{n-1} p_{i j}(t) e^{\alpha \tau_{i j}(t)}\right\}, \quad t \in[0, \omega] .
\end{aligned}
$$

Then problem (5.1), (5.3) is uniquely solvable for every summable $f=\operatorname{col}\left(f_{1}, \ldots, f_{n}\right)$ and $c=\left\{c_{1}, \ldots, c_{n}\right\} \in R^{n}$, and the elements of the nth row of Green's matrix of problem (5.1), (5.3) satisfy the inequalities: $G_{n j}(t, s) \leq 0$ for $t, s \in[0, \omega], j=1, \ldots, n, G_{n n}(t, s)<0$ for $0 \leq t<s \leq \omega$.

Proof. Repeating the explanations in the beginning of the proof of Theorem 4.1, we can obtain on the basis of Theorem 3.1 of the paper [13] that all the elements of $(n-1) \times(n-1)$ Green's matrix $K(t, s)$ of the auxiliary problem, consisting of the system

$$
x_{i}^{\prime}(t)+\sum_{j=1}^{n-1} p_{i j}(t) x_{j}\left(t-\tau_{i j}(t)\right)=f_{i}(t), \quad i=1, \ldots, n-1, t \in[0, \omega],
$$

and the boundary conditions $x_{i}(0)=x_{i}(\omega)+c_{i}, i=1, \ldots, n-1$, are nonnegative.

Let us set $v_{i}(t)=-e^{-\alpha t}$ for $i=1, \ldots, n-1$, and $v_{n}(t)=e^{-\alpha t}$ in the condition (1) of Theorem 3.1. We obtain that the condition (1) of Theorem 3.1 is satisfied if $\alpha$ satisfies the following system of the inequalities:

$$
\begin{gathered}
\alpha \leq-p_{i n}(t) e^{\alpha \tau_{i n}(t)}+p_{i i}(t)+\sum_{j=1, i \neq j}^{n-1} p_{i j}(t) e^{\alpha \tau_{i j}(t)}, \quad i=1, \ldots, n-1, t \in[0, \omega], \\
p_{n n}(t) e^{\alpha \tau_{n n}(t)}-\sum_{j=1}^{n-1} p_{n j}(t) e^{\alpha \tau_{n j}(t)} \leq \alpha, \quad t \in[0, \omega] .
\end{gathered}
$$

Now by virtue of Theorem 3.1, all elements of the $n$th row of Green's matrix of problem (5.1), (5.3) satisfy the inequalities $G_{n j}(t, s) \leq 0$ for $t, s \in[0, \omega], j=1, \ldots, n$, while $G_{n n}(t, s)<$ 0 for $0 \leq t<s \leq \omega$.

Remark 5.2. It was explained in the previous paragraph that in the case of ordinary system $\left(\tau_{i j}=0, i, j=1, \ldots, n\right)$ with constant coefficients $p_{i j}$, inequality (5.4) is best possible in a corresponding case. 
Let us consider the second-order scalar differential equation

$$
y^{\prime \prime}(t)+p_{11}(t) y^{\prime}(t)+p_{12}(t) y\left(t-\tau_{12}(t)\right)=f_{1}(t), \quad t \in[0, \omega],
$$

where $y(\xi)=y^{\prime}(\xi)=0$ for $\xi<0$, with the boundary conditions

$$
y^{\prime}(0)=y^{\prime}(\omega)+c_{1}, \quad y(\omega)=c_{2}
$$

and the corresponding differential system of the second order

$$
\begin{gathered}
x_{1}^{\prime}(t)+p_{11}(t) x_{1}(t)+p_{12}(t) x_{2}\left(t-\tau_{12}(t)\right)=f_{1}(t), \quad t \in[0, \omega], \\
x_{2}^{\prime}(t)-x_{1}(t)=0,
\end{gathered}
$$

where $x_{1}(\xi)=x_{2}(\xi)=0$ for $\xi<0$, with the boundary conditions

$$
x_{1}(0)=x_{1}(\omega)+c_{1}, \quad x_{2}(\omega)=c_{2} .
$$

It should be noted that the element $G_{21}(t, s)$ of Green's matrix of system (5.10), (5.11) coincides with Green's function $W(t, s)$ of the problem (5.8), (5.9) for scalar second-order equation.

Theorem 5.3. Assume that $p_{12} \geq 0$ and there exists a positive number $\alpha$ such that

$$
\alpha^{2}+p_{12}(t) e^{\alpha \tau_{12}(t)} \leq \alpha p_{11}(t), \quad t \in[0, \omega]
$$

Then problem (5.10), (5.11) is uniquely solvable for every summable $f=\operatorname{col}\left(f_{1}, f_{2}\right)$ and $c=\operatorname{col}\left(c_{1}, c_{2}\right) \in R^{2}$, and the elements of the second row of Green's matrix of this problem satisfy the inequalities: $G_{2 j}(t, s) \leq 0, j=1,2, t, s \in(0, \omega)$, while $G_{22}(t, s)<0$ for $0 \leq t<s<\omega$.

In order to prove Theorem 5.3, we set $v_{1}(t)=-\alpha e^{-\alpha t}, v_{2}(t)=e^{-\alpha t}$ in the assertion (1) of Theorem 3.1.

Remark 5.4. Inequality (5.12) is best possible in the following sense. Let us add $\varepsilon$ in its right hand side. We get that the inequality

$$
\alpha^{2}+p_{12}(t) e^{\alpha \tau_{12}(t)} \leq \alpha p_{11}(t)+\varepsilon, \quad t \in[0, \omega],
$$

and the assertion of Theorem 5.3 is not true. Let us set that coefficients are constants: $p_{11}=0$ and $0<p_{12}<\varepsilon$. It is clear that the inequality (5.13) is fulfilled if we set $\alpha$ small enough. Consider the following homogeneous boundary value problem:

$$
\begin{gathered}
x_{1}^{\prime}(t)+p_{12} x_{2}(t)=0, \\
x_{2}^{\prime}(t)-x_{1}(t)=0, \quad t \in[0, \omega], \\
x_{1}(0)=x_{1}(\omega), \quad x_{2}(\omega)=0 .
\end{gathered}
$$


The components $x_{1}, x_{2}$ of the solution vector are periodic and for $\omega=2 \pi / \sqrt{p_{12}}$ the boundary value problem (5.14) has a nontrivial solution.

Let us prove the following assertions, giving an efficient test of nonpositivity of the elements in the $n$th row of Green's matrix in the case when the coefficients $\left|p_{n j}\right|$ are small enough for $j=1, \ldots, n-1$.

Theorem 5.5. Let the following conditions be fulfilled:

(1) $p_{i j} \leq 0$ for $i \neq j, i, j=1, \ldots, n-1$;

(2) $p_{j n} \geq 0, p_{n j} \leq 0, p_{n n} \geq 0$ for $j=1, \ldots, n-1$;

(3) $\tau_{n n}=$ const $>0$, and other delays $\tau_{i j}$ are zeros;

(4) the inequalities

$$
\begin{gathered}
p_{n n}(t) \tau_{n n} \exp \left\{\tau_{n n} \sum_{j=1}^{n-1}\left|p_{n j}\right|^{*}\right\} \leq \frac{1}{e}, \quad t \in[0, \omega], \\
\frac{1}{\tau_{n n}}+\sum_{j=1}^{n-1}\left|p_{n j}\right|^{*} \leq \min _{1 \leq i \leq n-1}\left\{-p_{i n}(t)+\sum_{j=1, i \neq j}^{n} p_{i j}(t)\right\}, \quad t \in[0, \omega],
\end{gathered}
$$

are fulfilled.

Then problem (5.1), (5.3) is uniquely solvable for every summable $f=\operatorname{col}\left(f_{1}, f_{2}, \ldots, f_{n}\right)$ and $c=\left(c_{1}, c_{2}, \ldots, c_{n}\right) \in R^{n}$, and the elements of the nth row of its Green's matrix satisfy the inequalities: $G_{n j}(t, s) \leq 0$ for $j=1, \ldots, n$, while $G_{n n}(t, s)<0$ for $0<t<s<\omega$.

Proof. Let us set $v_{i}(t)=-e^{-\alpha t}$ for $i=1, \ldots, n-1$, and $v_{n}(t)=e^{-\alpha t}$ in the condition (1) of Theorem 3.1.

$$
p_{n n}(t) e^{\alpha \tau_{n n}}-\sum_{j=1}^{n-1} p_{n j}(t) \leq \alpha \leq \min _{1 \leq i \leq n-1}\left\{-p_{i n}(t)+\sum_{j=1, i \neq j}^{n} p_{i j}(t)\right\}, \quad t \in[0, \omega] .
$$

In the left-hand side, we have the inequality

$$
p_{n n}(t) e^{\alpha \tau_{n n}}-\sum_{j=1}^{n-1} p_{n j}(t) \leq \alpha, \quad t \in[0, \omega]
$$

which is fulfilled when

$$
p_{n n}(t) \leq\left\{\alpha-\sum_{j=1}^{n-1}\left|p_{n j}\right|^{*}\right\} e^{-\alpha \tau_{n n}}, \quad[0, \omega]
$$

The right-hand side in inequality (5.18) gets its maximum for $\alpha=1 / \tau_{n n}+\sum_{j=1}^{n-1}\left|p_{n j}\right|^{*}$. Substituting this $\alpha$ into (5.19) and the right part of (5.17), we obtain inequalities (5.15) and (5.16). 
Remark 5.6. It can be stressed that we do not require a smallness of the interval $[0, \omega]$ in Theorems 5.1-5.5.

Remark 5.7. It can be noted that inequality (5.15) is best possible in the following sense. If $p_{n j}=0$ for $j=1, \ldots, n-1, p_{n n}=$ const $>0$, then system (5.1) and inequality (5.15) become of the following forms:

$$
\begin{gathered}
x_{i}^{\prime}(t)=f_{i}(t), \quad i=1, \ldots, n-1, \quad x_{n}^{\prime}(t)+p_{n n} x_{n}\left(t-\tau_{n n}\right)=f_{n}(t), \quad t \in[0, \omega] . \\
p_{n n} \tau_{n n} \leq \frac{1}{e}, \quad t \in[0, \omega]
\end{gathered}
$$

respectively. The opposite to (5.21) inequality $p_{n n} \tau_{n n}>1 / e$ implies oscillation of all solutions [15] of the equation

$$
x_{n}^{\prime}(t)+p_{n n} x\left(t-\tau_{n n}\right)=0, \quad t \in[0, \omega]
$$

It implies that the homogeneous problem

$$
\begin{gathered}
x_{i}^{\prime}(t)=0, \quad i=1, \ldots, n-1, \quad x_{n}^{\prime}(t)+p_{n n} x_{n}\left(t-\tau_{n n}\right)=0, \quad t \in[0, \omega], \\
x_{i}(0)=x_{i}(\omega), \quad i=1, \ldots, n-1, \quad x_{n}(\omega)=0
\end{gathered}
$$

has nontrivial solutions for corresponding $\omega$. Now it is clear that we cannot substitute

$$
p_{n n}(t) \tau_{n n} \exp \left\{\tau_{n n} \sum_{j=1}^{n-1}\left|p_{n j}\right|^{*}\right\} \leq \frac{1+\varepsilon}{e}, \quad t \in[0, \omega]
$$

where $\varepsilon$ is any positive number instead of inequality (5.15).

\section{Acknowledgments}

The author thanks the referees for their available remarks. This research was supported by The Israel Science Foundation (Grant no. 828/07).

\section{References}

[1] N. V. Azbelev, V. P. Maksimov, and L. F. Rakhmatullina, Introduction to the Theory of Functional Differential Equations, vol. 3 of Advanced Series in Mathematics Science and Engineering, World Federation, Atlanta, Ga, USA, 1995.

[2] S. A. Tchaplygin, New Method of Approximate Integration of Differential Equations, GTTI, Moscow, UK, 1932.

[3] N. N. Luzin, "On the method of approximate integration of academician S. A. Tchaplygin," Uspekhi Matematicheskikh Nauk, vol. 6, no. 6, pp. 3-27, 1951 (Russian).

[4] V. Lakshmikantham and S. Leela, Differential and Integral Inequalities, Academic Press, 1969.

[5] M. A. Krasnosel'skii, G. M. Vainikko, P. P. Zabreiko, Ja. B. Rutitskii, and V. Ja. Stezenko, Approximate Methods for Solving Operator Equations, Nauka, Moscow, Russia, 1969. 
[6] I. Kiguradze and B. Puza, “On boundary value problems for systems of linear functional-differential equations," Czechoslovak Mathematical Journal, vol. 47, no. 2, pp. 341-373, 1997.

[7] I. Kiguradze and B. Puza, Boundary Value Problems for Systems of Linear Functional Differential Equations, vol. 12 of Folia Facultatis Scientiarium Naturalium Universitatis Masarykianae Brunensis. Mathematica, FOLIA, Masaryk University, Brno, Czech Republic, 2003.

[8] I. T. Kiguradze, "Boundary value problems for systems of ordinary differential equations," in Current Problems in Mathematics. Newest Results, vol. 30 of Itogi Nauki i Tekhniki, pp. 3-103, Akad. Nauk SSSR Vsesoyuz. Inst. Nauchn. i Tekhn. Inform., Moscow, Russia, 1987, English translated in Journal of Soviet Mathematics, vol. 43, no. 2, 2259-2339, 1988.

[9] T. Ważewski, "Systèmes des équations et des inégalités différentielles ordinaires aux deuxièmes membres monotones et leurs applications," Annales Polonici Mathematici, vol. 23, pp. 112-166, 1950.

[10] R. P. Agarwal and A. Domoshnitsky, "Non-oscillation of the first-order differential equations with unbounded memory for stabilization by control signal," Applied Mathematics and Computation, vol. 173 , no. 1, pp. 177-195, 2006.

[11] A. Domoshnitsky, "Maximum principles and nonoscillation intervals for first order Volterra functional differential equations," Dynamics of Continuous, Discrete $\mathcal{E}$ Impulsive Systems A, vol. 15, no. 6 , pp. 769-814, 2008.

[12] R. Hakl, A. Lomtatidze, and J. Sremr, Some Boundary Value Problems for First Order Scalar Functional Differential Equations, FOLIA, Masaryk University, Brno, Czech Republic, 2002.

[13] R. P. Agarwal and A. Domoshnitsky, "On positivity of several components of solution vector for systems of linear functional differential equations," Glasgow Mathematical Journal, vol. 52, no. 1, pp. 115-136, 2010.

[14] A. Domoshnitsky, "New concept in the study of differential inequalities," in Functional-Differential Equations, vol. 1 of Functional Differential Equations, Israel Seminar, pp. 52-59, The College of Judea \& Samaria, Ariel, Israel, 1993.

[15] I. Győri and G. Ladas, Oscillation Theory of Delay Differential Equations, Oxford Mathematical Monographs, The Clarendon Press, Oxford University Press, New York, NY, USA, 1991. 